\title{
Oesophageal cancer: risks, prevention, and diagnosis
}

An editorial error occurred in figure 2 of this clinical update by Thrumurthy and colleagues (BMJ 2019;366:14373, doi:10.1136/ bmj.14373). The colours for female cases and male cases were wrongly transposed from ages 60-64 upwards. The figure has been corrected online. 